\title{
An Improved UPLC Method for Determining Uric Acid in Rat Serum and Comparison Study with Commercial Colorimetric Kits
}

\author{
Shaoshi Wen ${ }^{1}$, Zixin Zhang ${ }^{2}$, Xiaopeng Chen ${ }^{2}$, Jinchang Liu ${ }^{2}$, Haiyang $\mathrm{Yu}^{2}$, Lifeng $\mathrm{Han}^{2}$, \\ Lijun Jin $^{1}$, Yi Zhang ${ }^{2 *}$ and Tao Wang ${ }^{*}$ \\ ${ }^{1}$ Tianjin State Key Laboratory of Modern Chinese Medicine, Tianjin University of Traditional Chinese Medicine, \\ 312 Anshanxi Road, Nankai District, Tianjin, 300193, China \\ ${ }^{2}$ Tianjin Key Laboratory of TCM Chemistry and Analysis, Institute of Traditional Chinese Medicine, Tianjin University \\ of Traditional Chinese Medicine, 312 Anshan Road, Nankai District, Tianjin, 300193, China
}

Received: 05 February 2018; accepted: 21 March 2018

\begin{abstract}
Uric acid (UA) is the final product of purine metabolism in humans. Elevated serum UA levels lead to the development of hyperuricemia, gout, kidney diseases, and metabolic syndrome. Accurate determination of UA plays a critical role in clinical diagnosis and laboratory investigation. An ultra-performance liquid chromatography (UPLC) with ultraviolet detection method has been developed and validated for UA analysis. Separation was achieved by a Waters ethylene bridged hybrid (BEH) Amide column $(50 \mathrm{~mm} \times 2.1 \mathrm{~mm}$ i.d., $1.7 \mu \mathrm{m})$ with acetonitrile and $0.1 \%$ acetic acid in deionized water in the proportion of 90 to $10(\mathrm{v} / \mathrm{v})$ as the mobile phase. The limit of detection and limit of quantification were 0.09 and $0.18 \mu \mathrm{mol} / \mathrm{L}$, respectively. The method was validated by evaluating recovery $(98.37-104.20 \%)$, accuracy $(0.47-0.90 \%)$, and precision $(1.24-1.81 \%$ for intra-batch and $1.76-3.98 \%$ for inter-batch). This method was then applied to UA determination in rat serum of hyperuricemia model. The results from UPLC, high-performance liquid chromatography (HPLC), and uric acid kits (phosphor-tungstic acid-based kit and uricase-based kit) were compared. The UPLC results were in very good agreement with HPLC. The developed method could be employed as a useful tool for the determination of UA in biofluids.
\end{abstract}

Keywords: Uric acid, UPLC, colorimetric kit, rat serum, hyperuricemia

\section{Introduction}

Uric acid (UA) is the final product of purine nucleosides catabolization [1]. Elevated serum UA levels are associated with risk of hyperuricemia, gout, and kidney-related diseases [2]. Thus, plasma UA has been used as a biomarker in physiological investigation and disease diagnosis.

Some methods have been developed and applied to determine the levels of UA in the blood and urine samples [3-6]. Uric acid kits are commonly used in most of the clinical analysis [7-9]. The enzymic method with uricase $[7,10]$ and colorimetric procedure with phosphotungstate [11] are two reaction mechanisms of uric acid kit (Figure 1). In uricase-based kit, UA is enzymatically oxidized by oxygen in the presence of uricase, generating allantoin, $\mathrm{CO}_{2}$, and hydrogen peroxide [12-15]. The hydrogen peroxide further reacts with reducing agent to form a colored dye complex. The shade of the dye complex is directly proportional to the amount of UA contained in the sample. In the phosphor-tungstic acid-based kit, the oxidizing agent is used as color reagent to generate chromogenic product that could be determined by colorimetric analysis. It is important to note that commercial uric acid kits are generally validated for UA analysis of human serum, but not for other matrices. Some mammals, such as rat, metabolize urate to allantoin by endogenous uricase, causing uncertainty in UA levels determination. Meanwhile, the reduction substances [16] contained in the bio-samples could influence the generation of chromogenic product $[11,17]$, which would cause biases on the determination results.

\footnotetext{
* Authors for correspondence: zhwwxzh@263.net (Y.Z.) or wangtao@tjutcm.
} edu.cn (T.W.); +86225959 6163; +862259596163

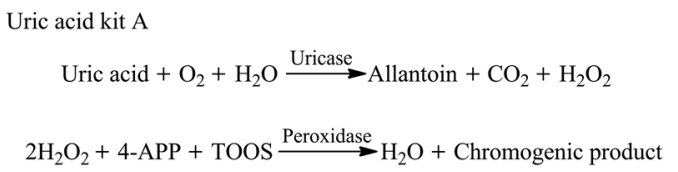

Figure 1. Mechanism of commercial uric acid kits (uric acid kit A: uricase-based kit; uric acid kit B: phosphor-tungstic acid-based kit; 4-AAP: 4-aminoantipyrine; TOOS: $N$-ethyl- $N$-(2-hydroxy-3-sulfopropyl)m-toluidine sodium salt)

High-performance liquid chromatography (HPLC) was developed as quantitative method for UA determination in complex matrices, especially used for laboratory hyperuricemic animal model. However, for the high solubility and polarity of UA, the HPLC separation in many cases requires highly aqueous mobile phase to keep retention time and resolution, which is unsuitable for most chromatographic columns.

In the past decade, ultra-performance liquid chromatography (UPLC) is of high sensitivity, short analysis time, and good resolution, which has been successfully implemented to overcome some of the analytical difficulties recognized with endogenous interference [18-20]. No publication reports the determination on UA specifically besides few papers dealing with the multicompounds including UA in biofluids using UPLC. Ma et al. [21] reported determination of UA and creatinine in serum and urine of hyperuricemic mice by injecting directly to MS/MS using the guard column. The peak shape illustrates the ability of

This is an open-access article distributed under the terms of the Creative Commons Attribution-NonCommercial 4.0 International License (https://creativecommons.org/licenses/by-nc/4.0/), which permits unrestricted use, distribution, and reproduction in any medium for non-commercial purposes, provided the original author and source are credited, a link to the CC License is provided, and changes - if any - are indicated. 
the guard column to remove interference and enrich the target compounds is limited. Boelaert et al. [22] studied seven uremic retention toxins of chronic kidney disease patients by UPLC-tandem mass spectrometry (MS/MS), which need a relatively long analytical time for the whole method. Meanwhile, in reversephase liquid chromatography, the volume of aqueous phase contained in the mobile phase for the separation of UA was much higher than other metabolites [23], resulting in poor reservation on $\mathrm{C} 18$ column and low efficiency of ionization on MS ion source. Therefore, it is necessary to develop a quick, sensitive, reliable, and precise method for determination of UA in biofluids specifically using UPLC.

In this work, we describe the development and application of a UPLC-ultraviolet (UV) method for detection and quantification of UA in rat serum. Ethylene bridged hybrid (BEH) Amide column is used to gain good reservation of UA. This method was applied to the determination of UA in hyperuricemic rat serum samples. A conventional HPLC-UV method was also developed here. The results of UPLC-UV, HPLC-UV, and commercial colorimetric kits measurements for serum uric acid were compared. Our findings confirm that the UPLC-UV method provides a quick, sensitive, and reliable approach for quantifying UA in biofluids.

\section{Experimental}

Chemicals and Materials. Uric acid kit A (uricase-based) was obtained from Biosino Bio-Technology Co., Ltd. (Beijing, China). Uric acid kit B (phosphor-tungstic acid-based) was obtained from Nanjing Jiancheng Biological Technology Co., Ltd. (Nanjing, China). Uric acid sodium salt and potassium oxonate were purchased from Sigma-Aldrich (MO, USA). Probenecid was purchased from Alfa-Aesar (Lancashire, UK). Disodium hydrogen phosphate was purchased from Guangfu Technology Development Co., Ltd. (Tianjin, China). Acetonitrile and methanol (Fisher Scientific, Waltham, USA) were HPLC grade. Ultrapure water was obtained using a Millipore Milli-Q Academic ultra-pure water system (MA, USA).

Potassium Oxonate-Induced Hyperuricemia Rats. All experimental protocols involving animals were reviewed and approved by the Ethics Committee of Tianjin University of Traditional Chinese Medicine. Sprague-Dawley (SD) rats (250$300 \mathrm{~g}$ ) were purchased from HFK Bioscience Co., Ltd. (Beijing, China). The rats were housed in a specific pathogen free (SPF) zone at a constant temperature of $23 \pm 2{ }^{\circ} \mathrm{C}$ with a 12-h light/ dark cycle to acclimatize before the experiments. The animals were fed a standard commercial rodent diet and tap water ad libitum. According to the previous report [24], potassium oxonate, the uricase inhibitor, was used to induce hyperuricemia in rats. Briefly, SD rats were randomly distributed into 2 groups (6 rats per group): control group and potassium oxonate-induced hyperuricemia group. The animals of hyperuricemia group were administered orally with potassium oxonate solution $5 \%$ acacia solution solutions $(200 \mathrm{mg} / \mathrm{kg})$, while the control group had an equal volume of $5 \%$ acacia solution. Blood samples (ca. $0.5 \mathrm{~mL}$ ) were collected from infraorbital venous plexus of rats under ether anesthesia at $1 \mathrm{~h}$ and $2 \mathrm{~h}$ later.

Serum Sample Preparation. The blood sample was collected in Eppendorf tube and centrifuged for $10 \mathrm{~min}$ at $5000 \mathrm{~g}$. After centrifuging, the supernatant was divided into two parts. One part was directly used for uric acid kit analysis, and the other part needs further pretreatment for chromatography analysis. The further pretreatment was established by adapting literature method [25]. In brief, serum $(20 \mu \mathrm{L})$ was added to $180 \mu \mathrm{L}$ perchloric acid $(0.3 \mathrm{M})$ and placed in ice-water bath for $30 \mathrm{~min}$ after vortexing. The mixture was centrifuged for $10 \mathrm{~min}$ at $10,000 \mathrm{~g}$ under $4{ }^{\circ} \mathrm{C}$, and the supernatant was neutralized with $0.8 \mathrm{M}$ $\mathrm{Na}_{2} \mathrm{HPO}_{4}$ solution. Equal volume acetonitrile was added, and the mixture was centrifuged for $10 \mathrm{~min}$ at $10,000 \mathrm{~g}$ under $4{ }^{\circ} \mathrm{C}$. The supernatant was used for liquid chromatography injection.

Uric Acid Kits Analysis. Uric acid kit A (uricase-based) and uric acid kit B (phosphor-tungstic acid-based) were used to determine rat serum sample followed manufacturer's instructions. Briefly, for uric acid kit A, $20 \mu \mathrm{L}$ serum sample was added to $200 \mu \mathrm{L}$ reaction solution (contains $600 \mathrm{U} / \mathrm{L}$ uricase, $1800 \mathrm{U} / \mathrm{L}$ peroxidase, $0.3 \mathrm{mM}$ 4-aminoantipyrine [4-AAP], $1.4 \mathrm{mM} \mathrm{N}$ ethyl- $N$-(2-hydroxy-3-sulfopropyl)-3-methylaniline [TOOS] in $100 \mathrm{mM}$ phosphate buffer [pH 7.8]). After 30 min incubation at $37^{\circ} \mathrm{C}$, the absorbance was measured at $\mathrm{OD}_{550 \mathrm{~nm}}$ by Multiskan MK3 microplate reader (Thermo Fisher Scientific, Waltham, USA). For uric acid kit B, $20 \mu \mathrm{L}$ serum sample was mixed with $200 \mu \mathrm{L}$ tungstic acid to precipitate serum proteins at room temperature for $10 \mathrm{~min}$. Then, the mixture was centrifuged for $5 \mathrm{~min}$ at $3000 \mathrm{~g}$ under $4{ }^{\circ} \mathrm{C}$ and the supernatant was added to $100 \mu \mathrm{L}$ phospho-tungstic acid solution to format tungsten blue by UA. The absorbance was measured at $\mathrm{OD}_{690} \mathrm{~nm}$ by Multiskan MK3 microplate reader (Thermo Fisher Scientific, Waltham, USA).

Chromatographic Procedures. The UPLC analysis was performed using a Waters ACQUITY UPLC system (Waters, Milford, USA) including ACQUITY UPLC H-CLASS pump, auto sampler, degasser, and hp system controller (hp pro, USA). The chromatograph was fitted with a BEH Amide column (50 mm $\times 2.1 \mathrm{~mm}$ i.d., $1.7 \mu \mathrm{m}$, Waters Co. Ltd., Milford, USA). Mobile phase consisted of acetonitrile and $0.1 \%$ acetic acid in deionized water in proportion of 90 to $10(\mathrm{v} / \mathrm{v})$. The column was maintained at $30{ }^{\circ} \mathrm{C}$, and the flow rate was $0.3 \mathrm{~mL} / \mathrm{min}$. UV detection was performed at $284 \mathrm{~nm}$. The injection volume was $2 \mu \mathrm{L}$.

The HPLC analysis was performed using a Waters ACQUITY HPLC system (Waters, Milford, USA) including Waters e2695 pump, Waters e2695 auto sampler, Waters 2998 UV detector, Waters e2695 degasser, and Dell system controller (Optiplex 7010). Analysis parameter was set as literature with a little modification [26]. The chromatograph was fitted with a COSMOSIL C18 column $(150 \mathrm{~mm} \times 4.6 \mathrm{~mm}$ i.d., $5 \mu \mathrm{m}$, Nacalai Tesque Co. Ltd. Japan). Mobile phase consisted of methanol and $74 \mathrm{mM}$ phosphate buffer in proportion of 2 to $98(\mathrm{v} / \mathrm{v})$. The column was maintained at $25^{\circ} \mathrm{C}$, and the flow rate was $1.0 \mathrm{~mL} / \mathrm{min}$. UV detection was performed at $284 \mathrm{~nm}$. The injection volume was $30 \mu \mathrm{L}$.

Method Validation. The method was validated by testing linearity, sensitivity (limits of detection and limits of quantification), accuracy, precision, stability, and recovery.

(a) Linearity and Sensitivity. Standard UA solutions were prepared using reagent-grade chemicals at time of use. To prepare the calibration curves, a series of calibration standards of UA was obtained by diluting with water at appropriate concentrations. Calibration curves were created by plotting peak area of UA against the concentrations and fitting these data with linear regression. The limit of detection (LOD) and limit of quantification (LOQ) were determined at a signal-to-noise ratio of $3: 1$ and $10: 1$ by analyzing the UA standard solutions for UPLC and HPLC. The LOD of uric acid kits was determined as the mean plus 3 fold of standard deviation of blank samples. The LOQ of uric acid kits was achieved at least five times the response compared to blank response and should have precision that does not exceed $20 \%$ of the coefficient of variation and accuracy within $20 \%$ of the nominal concentration.

(b) Accuracy. To evaluate the accuracy, the prepared samples (sample A: $20 \mathrm{mg} / \mathrm{L}$ UA standard sample $+200 \mu \mathrm{L}$ rat serum sample; sample B: $20 \mathrm{mg} / \mathrm{L}$ UA standard sample) were determined by uric acid kit methods (uric acid kit A and uric acid kit B) and chromatographic methods (HPLC and UPLC). The accuracy was estimated by comparing the measured concentration 
with its true value, and the relative error (RE, \%) was used to evaluate the accuracy.

(c) Precision. To evaluate the precision, the prepared sample (sample A: $20 \mathrm{mg} / \mathrm{L}$ UA standard sample $+200 \mu \mathrm{L}$ rat serum sample; sample B: $20 \mathrm{mg} / \mathrm{L}$ UA standard sample; sample C: $16 \mathrm{mg} / \mathrm{L}$ UA standard sample; sample D: $8 \mathrm{mg} / \mathrm{L}$ UA standard sample) were determined by uric acid kit methods (uric acid kit A and uric acid kit B) and chromatographic methods (HPLC and UPLC). The intra-batch precision was based on samples analyzed in one day, while the inter-batch precision was based on samples analyzed in each of 3 days. Relative standard deviation (RSD, \%) was used to assess the intrabatch and inter-batch precision.

(d) Stability of Uric Acid in Rat Serum Sample. The stability of UA was assessed by analyzing hyperuricemia group of rat serum sample at room temperature for $0-12 \mathrm{~h}$. RSD (\%) was used to assess the stability.

(e) Recovery. The recovery of UA was calculated by comparing peak areas in extracted serum samples with those in postextracted spiked samples at the same theoretical concentration.

\section{Results and Discussion}

Method Performance. Table 1 summarizes the analytical performances of uric acid kits, HPLC, and UPLC, including linear range, regression equation, correlation coefficient $(R)$, limit of detection (LOD), and limit of quantification (LOQ)

The linear calibration range studied was $12.11-1550.05 \mu \mathrm{mol} / \mathrm{L}$ for uric acid kit A, 24.22-775.03 $\mu \mathrm{mol} / \mathrm{L}$ for uric acid kit B, $0.73-187.50 \mu \mathrm{mol} / \mathrm{L}$ for HPLC, and $0.18-750.00 \mu \mathrm{mol} / \mathrm{L}$ for UPLC. All methods possessed good correlation coefficient above 0.9970 . The RSDs for reproducibility were less than $1.73 \%$, indicating good reproducibility of the methods. The LODs were 12.11, 24.22, 0.18, and $0.09 \mu \mathrm{mol} / \mathrm{L}$ for uric acid kit $\mathrm{A}$, uric acid kit B, HPLC, and UPLC. Compared with uric acid kits, the liquid chromatography methods are more sensitive, which make the methods gain wider range of applications, especially on low content biofluid sample. Meanwhile, the LOD of UPLC-UV observed in this study, which is $0.09 \mu \mathrm{mol} / \mathrm{L}$, were equivalent [27] or more sensitive [28] compared to the previously published mass spectrometry methods for serum uric acid determination.

The accuracy and precision of the methods are presented in Table 2. All methods possessed good accuracy for standard sample in pure solvent (sample B, $-4.56 \%$ to $8.73 \%$ ). However, for the UA in rat serum sample (sample A), the accuracies were $-23.21 \%, 19.86 \%, 2.23 \%$, and $0.47 \%$ for uric acid kit A, uric acid kit B, HPLC, and UPLC, respectively. Compared with uric acid kit methods, the chromatographic methods shown good accuracy to determined UA in rat serum sample.

The intra-batch precision was $1.24-4.55 \%$ for rat serum sample (sample A) and $0.57-2.78 \%$ for standard sample (samples B, $\mathrm{C}$, and D). The inter-batch precision was $3.00-5.67 \%$ for rat serum sample (sample A) and $1.76-4.11 \%$ for standard sample (samples B, C, and D). Though the upper range of rat serum sample is higher than the standard sample, all precisions are acceptable for biological sample analysis.

To check for bench-top stability, the rat serum samples were analyzed throughout the $12 \mathrm{~h}$ period, and the RSDs were $3.82 \%$ and $3.11 \%$ for UPLC and HPLC, respectively. These findings suggest that UA are stable in rat serum sample over a $12 \mathrm{~h}$ period at room temperature.

Recoveries were also tested in the rat serum samples. The concentration of the UA in rat serum sample was 24.2 and $26.3 \mathrm{mg} / \mathrm{L}$ (142.9 and $153.6 \mu \mathrm{mol} / \mathrm{L}$ ) for HPLC and UPLC, respectively. As listed in Table 3, the recoveries were in the range of 98.45$103.27 \%$ for HPLC and $98.37-104.20 \%$ for UPLC. The results indicated that the serum extraction method works well.

Method Application. In this work, UPLC, HPLC, uric acid kit $\mathrm{A}$, and uric acid kit $\mathrm{B}$ have been applied to determine the UA

Table 3. Recoveries of HPLC and UPLC

\begin{tabular}{lcccc}
\hline Methods & $\begin{array}{c}\text { Extracted sample } \\
\text { UA area } \\
\text { Mean } \pm \text { SD }\end{array}$ & $\begin{array}{c}\text { Post-extracted } \\
\text { spiked sample } \\
\text { UA area }\end{array}$ & $\begin{array}{c}\text { Recovery } \\
(\%)\end{array}$ & $\begin{array}{c}\text { RSD (\%) } \\
(n=5)\end{array}$ \\
\hline HPLC & $253,707 \pm 2857$ & 252,504 & $98.45-103.27$ & 2.50 \\
UPLC & $26,589 \pm 269$ & 26,695 & $98.37-104.20$ & 2.30 \\
SD, standard deviation; RSD, relative standard deviation. & \\
\hline
\end{tabular}

Table 1. Analytical performances of uric acid kits, HPLC, and UPLC

\begin{tabular}{|c|c|c|c|c|c|}
\hline \multirow[t]{2}{*}{ Methods } & \multicolumn{3}{|c|}{ Linearity } & \multirow{2}{*}{$\begin{array}{c}\text { LOD } \\
(\mu \mathrm{mol} / \mathrm{L})\end{array}$} & \multirow{2}{*}{$\begin{array}{c}\mathrm{LOQ} \\
(\mu \mathrm{mol} / \mathrm{L})\end{array}$} \\
\hline & Range $(\mu \mathrm{mol} / \mathrm{L})$ & Regression equation & $R$ & & \\
\hline Uric acid kit $\mathrm{A}$ & $12.11-1550.05$ & $y=1188 x-71.9$ & 0.9995 & 12.11 & 24.22 \\
\hline Uric acid kit B & $24.22-775.03$ & $y=5394 x-221.3$ & 0.9970 & 24.22 & 48.44 \\
\hline HPLC & $0.73-187.50$ & $y=16,701 x+7724$ & 0.9995 & 0.18 & 0.73 \\
\hline UPLC & $0.18-750.00$ & $y=2074 x+305$ & 0.9999 & 0.09 & 0.18 \\
\hline
\end{tabular}

Table 2. Precision and accuracy for determination of UA standard samples and UA in hyperuricemia serum sample

\begin{tabular}{|c|c|c|c|c|}
\hline \multirow[t]{2}{*}{ Sample } & \multirow[t]{2}{*}{ Method } & \multirow[t]{2}{*}{ Accuracy (RE, \%) } & \multicolumn{2}{|c|}{ Precision (RSD, \%) } \\
\hline & & & Intra-batch $(n=6)$ & Inter-batch $(n=3)$ \\
\hline \multirow[t]{4}{*}{ Sample A } & Uric acid kit $\mathrm{A}$ & -23.21 & 3.66 & 5.67 \\
\hline & Uric acid kit B & 19.86 & 4.55 & 4.80 \\
\hline & HPLC & 2.23 & 1.78 & 3.00 \\
\hline & UPLC & 0.47 & 1.24 & 3.98 \\
\hline \multirow[t]{4}{*}{ Sample B } & Uric acid kit A & -4.56 & 2.78 & 1.92 \\
\hline & Uric acid kit B & 8.73 & 2.18 & 4.11 \\
\hline & HPLC & 0.07 & 0.57 & 3.61 \\
\hline & UPLC & 0.90 & 1.81 & 1.76 \\
\hline \multirow[t]{2}{*}{ Sample C } & Uric acid kit $A$ & - & 1.11 & - \\
\hline & Uric acid kit B & - & 1.73 & - \\
\hline \multirow[t]{2}{*}{ Sample D } & HPLC & - & 1.64 & - \\
\hline & UPLC & - & 0.75 & - \\
\hline
\end{tabular}

Sample A: 20 mg/L UA standard sample + $200 \mu \mathrm{L}$ hyperuricemia serum sample; sample B: 20 mg/L UA standard sample; sample C: 16 mg/L UA standard sample; sample D: $8 \mathrm{mg} / \mathrm{L}$ UA standard sample.

RE, relative error; RSD, relative standard deviation; -, not available. 
in rat serum sample. The chromatograms of HPLC and UPLC were shown in Figure 2. The UPLC could finish UA analysis in $4 \mathrm{~min}$, which is much shorter than published UPLC method using gradient elution in $15 \mathrm{~min}$ [22]. The differences between uric acid kit methods and chromatographic methods were investigated. As shown in Figure 3, the UA levels were detected in normal group (control) and hyperuricemia group on $1 \mathrm{~h}$ and $2 \mathrm{~h}$, respectively. Compared to the normal group, the levels of UA of hyperuricemia group raised 2.5 and 3 times on $1 \mathrm{~h}$ and $2 \mathrm{~h}$, which means that the hyperuricemia model on rats was successfully accomplished.

Method Comparison. The significance of the UPLC method is demonstrated by short analysis time, good accuracy and low parts-per-trillion detection limits. In this study, the results of UPLC and HPLC were in good agreement. In reported liquid chromatography (LC)-MS methods [27-29], reversed-phase C18 columns were commonly used in UA analysis methods. Due to the solubility and polarity of UA, the mobile phase requires high aqueous proportion, which is unsuitable for most of the reversedphase C18 columns and could lead to low efficiency of ionization on MS ion source. In this study, BEH Amide column was used in UPLC method to ensure $90 \%$ acetonitrile in mobile phase which enlarges the potential of further application in MS. The HPLC method with a COSMOSIL C18 column developed here was used as a reference method similar to published articles to check the consistency of UPLC and HPLC results.

Compared with UPLC and HPLC, UA levels analyzed by uricase-based kit were significantly lower, whereas the phosphortungstic acid-based kit showed two-folder higher. Generally, rats and mice were most commonly used for hyperuricemia
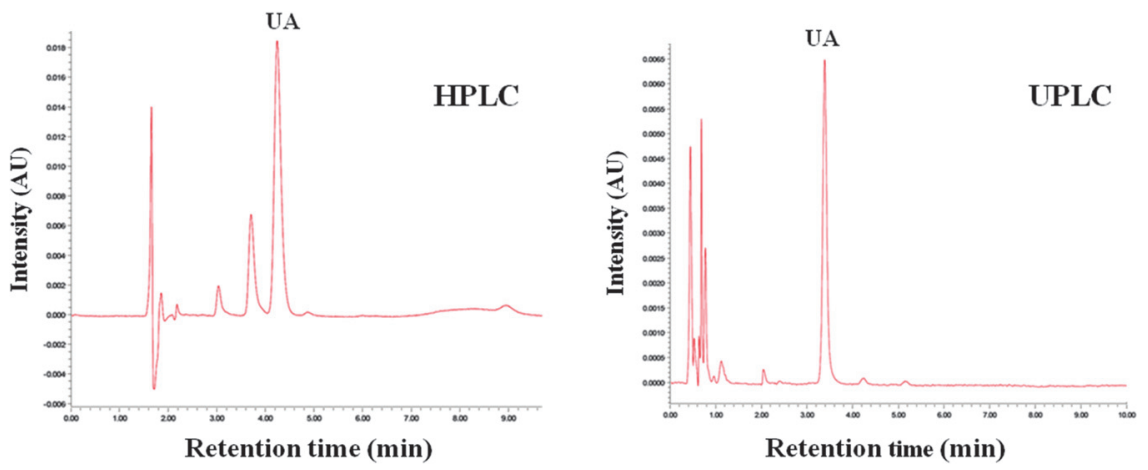

Figure 2. Chromatograms of uric acid (UA) from hyperuricemia group serum sample determined by HPLC and UPLC
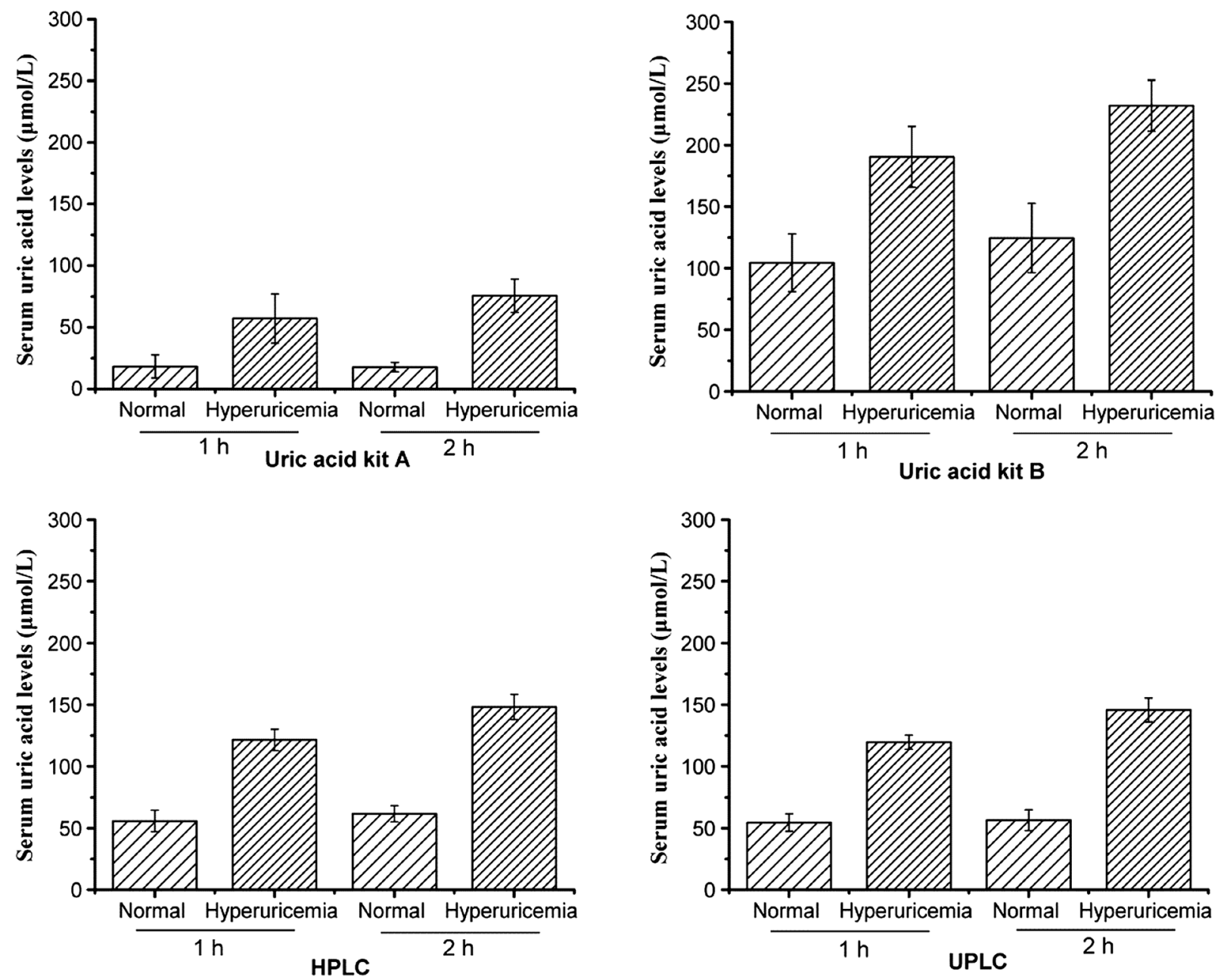

Figure 3. Comparison of UA levels detected by uric acid kit A (uricase-based kit), uric acid kit B (phosphor-tungstic acid-based kit), HPLC, and UPLC 
researching laboratory investigation. Different research groups reported control rat serum UA levels range from 75 to $160 \mu \mathrm{mol} / \mathrm{L}$ in rats [30-32] and 120 to $300 \mu \mathrm{mol} / \mathrm{L}$ in mice [30, 33-35] by using uric acid kit. In publications, uricasebased or phosphor-tungstic acid-based kit, which was developed for human diagnosis in clinic, was used in rodent serum UA level analysis [36, 37]. Uricase-based kit was developed based on the formation of sensitive chromogen by hydrogen peroxide. However, uricase is widely distributed in rodent animal serum, which could lead unexpected bias. Besides this, after protein precipitation, the rodent serum sample still contains trace antioxidant enzymes (catalase and superoxide dismutase) and diverse reducing substances (vitamin E, vitamin $\mathrm{C}$, and bilirubin), which could quench hydrogen peroxide and lead the determination results lower than the true value. Phosphor-tungstic acidbased kit, using phosphor-tungstic acid as color reagent, reacts with UA to form tungsten blue. In reaction process, serum amino acids, enzymes, and other reducing substances could increase the content of chromogenic product, leading test results higher than true value. Though uricase-based and phosphortungstic acid-based kits show different sensitivities to the interferences [38, 39], their easy application for automated analysis and favorable cost are incomparable advantages. To date, uric acid kits are preferable method for uric acid determination of physiological investigation and disease diagnosis in clinic.

\section{Conclusion}

A UPLC method for parts-per-trillion level quantitative analysis of UA in rat serum has been developed and validated. The key features of this method are short chromatographic run time (4 min), good accuracy, and wide linear range. The method was applied to rat serum UA determination in laboratory hyperuricemia investigation. This method could serve as a key method for UA determination in laboratory investigation.

Acknowledgments. The research was supported by National Natural Science Foundation of China (81173524 and 81673703), Important Drug Development Fund, Ministry of Science and Technology of China (2017ZX09305-002), and the Science and Technology Program of Tianjin, China (15PTCYSY00030 and 16ZXHLGX00170)

\section{References}

1. Balis, M. E. Adv. Clin. Chem. 1976, 213-246.

2. Xia, X.; Luo, Q.; Li, B.; Lin, Z.; Yu, X.; Huang, F. Metabolism 2016, $65,1326$.

3. Ozcan, A.; Sahin, Y. Biosens. Bioelectron. 2010, 25, 2497.
4. Alipour, E.; Majidi, M. R.; Saadatirad, A.; Golabi, S. M.; Alizadeh, A. M. Electrochim. Acta 2013, 91, 36.

5. Mazloum-Ardakani, M.; Rajabzadeh, N.; Dehghani-Firouzabadi, A.; Benvidi, A.; Mirjalili, B. B. F.; Zamani, L. J. Electroanal. Chem. 2016, 760, 151. 6. Raj, M. A.; John, S. A. Anal. Chim. Acta 2013, 771, 14.

7. Jalal, D. I.; Rivard, C. J.; Johnson, R. J. Eur. Renal Assoc. 2010, 25, 1865. 8. Tsioufis, C.; Kyvelou, S.; Dimitriadis, K.; Syrseloudis, D.; Sideris, S.;

Skiadas, I.; Katsi, V.; Stefanadi, E.; Lalos, S.; Mihas, C.; Poulakis, M.; Stefanadis, C. J. Hypertens. 2011, 25, 554.

9. Latha, R. C.; Daisy, P. Chem. Biol. Interact. 2011, 189, 112.

10. Jalal, D. I.; Jablonski, K. L.; Mcfann, K.; Chonchol, M. B.; Seals, D. R. Am. J. Hypertens. 2012, 25, 407.

11. Tan, Q. Y.; Wang, N.; Yang, H.; Chen, L.; Xiong, H. R.; Zhang, L. K.; Liu, J.; Zhao, C. J.; Zhang, J. Q. Drug Deliv. 2010, 17, 28.

12. Schrenkhammer, P.; Wolfbeis, O. S. Biosens. Bioelectron. 2008, 24, 1000.

13. Jin, D.; Seo, M. H.; Huy, B. T.; Pham, Q. T.; Conte, M. L.; Thangadurai, D.; Lee, Y. I. Biosens. Bioelectron. 2016, 77, 359

14. Zhao, Y.; Yang, X.; Lu, W.; Liao, H.; Liao, F. Microchim. Acta 2008, 164,1 .

15. Fischer, F.; Graschew, G.; Sinn, H. J.; Maierborst, W.; Lorenz, W. J.; Schlag, P. M. Clin. Chim. Acta 1998, 274, 89

16. Wilson, I. D.; Plumb, R.; Granger, J.; Major, H.; Williams, R.; Lenz, E. M. J. Chromatogr. B 2005, 817, 67.

17. Pileggi, V. J.; Di, G. J.; Wybenga, D. R. Clin. Chim. Acta 1972, 37, 141.

18. Krishnaiah, C.; Reddy, A. R.; Kumar, R.; Mukkanti, K. J. Pharm. Biomed. Anal. 2010, 53, 483 .

19. Shi, Z.; Hu, J.; Li, Q.; Zhang, S.; Liang, Y.; Zhang, H. J. Chromatogr. A 2014, 1355, 219-227.

20. Pretorius, C. J.; Mcwhinney, B. C.; Sipinkoski, B.; Johnson, L. A.;

Rossi, M.; Campbell, K. L.; Ungerer, J. P. J. Clin. Chim. Acta 2013, 419, 122.

21. Ma, Q. S.; Wang, Q.; Zhao, K. S.; Zhai, S. B.; Liu, S.; Liu, Z. Q. Chem. J. Chinese U. 2013, 34, 2716.

22. Boelaert, J.; Lynen, F.; Glorieux, G.; Eloot, S.; Van Landschoot, M.;

Waterloos, M. A.; Sandra, P.; Vanholder, R. Anal. Bioanal. Chem. 2013, 405, 1937. 23. Zhao, J. Anal. Methods 2013, 5, 6781.

24. Wang, M.; Liu, Y.; Yang, Y.; Zhang, D.; Kong, L. Eur. J. Pharmacol. 2015, 747, 59

25. Sa, M.; Ying, L.; Tang, A. G.; Xiao, L. D.; Ren, Y. P. Clin. Chim. Acta 2012, 413, 973 .

26. Tanaka, R.; Miyata, Y.; Sakazaki, F. Gout and Nucleic Acid Metab. 2013, 37, 117

27. Dai, X.; Fang, X.; Zhang, C.; Xu, R.; Xu, B. J. Chromatogr. B 2007, $857,287$.

28. Zhong, H. F.; Liang, Q. L.; Xia, J. F.; Hu, P.; Wang, Y. M.; Tong, X. L.; Luo, G. N. Chromatographia 2011, 73, 149.

29. George, S. K.; Dipu, M. T.; Mehra, U. R.; Singh, P.; Verma, A. K.; Ramgaokar, J. S. J. Chromatogr. B 2006, 832, 134.

30. Chen, G. L.; Wei, W.; Xu, S.-Y. Am. J. Chinese Med. 2006, 34, 77.

31. Hu, Q. H.; Zhang, X.; Wang, X.; Jiao, R. Q.; Kong, L. D. Eur. J. Nutr. 2011, 51, 593 .

32. Kou, Y.; Li, Y.; Ma, H.; Li, W.; Li, R.; Dang, Z. J. Tradit. Chin. Med. 2016, 36, 205

33. Li-Na, H.; Wei, W.; Chun-Yu, Z. Molecules 2015, 20, 17848.

34. Niu, Y.; Lu, W.; Gao, L.; Lin, H.; Liu, X.; Li, L. Pharm. Biol. 2012, $50,1177$.

35. Wang, J.; Pan, Y.; Hong, Y.; Zhang, Q. Y.; Wang, X. N.; Kong, L. D. Evid-Based Compl. Alt. 2012, 1.

36. Cheng, T. H.; Lin, J. W.; Chao, H. H.; Chen, Y. L.; Chen, C. H.; Chan, P.; Liu, J. C. Int. J. Cardiol. 2010, 139, 42.

37. Huang, J.; Wang, S.; Zhu, M.; Chen, J.; Zhu, X. Food Chem. Toxicol. 2011, 49, 1943.

38. Watts, R. W. E. Ann. Clin. Biochem. 1974, 11, 103.

39. Poole, R. A.; Kielar, F.; Richardson, S. L.; Stenson, P. A.; Parker, D. Chem. Commun. 2006, 39, 4084 